\title{
Magnetars: neutron stars with huge magnetic storms
}

\author{
Nanda Rea \\ Institut de Ciències de l'Espai (CSIC-IEEC), Campus UAB, Facultat de Ciències, \\ Torre C5-parell, E-08193 Barcelona, Spain \\ email: rea@ice.csic.es
}

\begin{abstract}
Among the many different classes of stellar objects, neutron stars provide a unique environment where we can test (at the same time) our understanding of matter with extreme density, temperature, and magnetic field. In particular, the properties of matter under the influence of magnetic fields and the role of electromagnetism in physical processes are key areas of research in physics. However, despite decades of research, our limited knowledge on the physics of strong magnetic fields is clear: we only need to note that the strongest steady magnetic field achieved in terrestrial labs is some millions of Gauss, only thousands of times stronger than a common refrigerator magnet. In this general context, I will review here the state of the art of our research on the most magnetic objects in the Universe, a small sample of neutron stars called magnetars. The study of the large high-energy emission, and the flares from these strongly magnetized ( $\sim 10^{15}$ Gauss) neutron stars is providing crucial information about the physics involved at these extremes conditions, and favoring us with many unexpected surprises.
\end{abstract}

Keywords. stars: neutron, X-rays: stars, stars: magnetic fields, stars: flare, dense matter, methods: data analysis

\section{Introduction}

Neutron stars are the debris of the supernova explosion of massive stars, the existence of which was first theoretically predicted around 1930 (Chandrasekhar 1931; Baade \& Zwicky 1934) and then observed for the first time more than 30 years later (Hewish et al. 1968). They were predicted all along as very dense and degenerate stars holding about 1.4 solar masses in a sphere of $10 \mathrm{~km}$ radius. We now know many different flavors of these compact objects, and many open questions are still waiting for an answer after decades of studies. The neutron star population is dominated by radio pulsars (thousands of objects), however in the last decades several extreme and puzzling sub-classes of isolated neutron stars were discovered: Anomalous X-ray Pulsars (AXPs), Soft Gamma Repeaters (SGRs; see Mereghetti 2008), Rotating Radio Transients (RRATs; Keane \& McLaughlin 2011), X-ray Dim Isolated Neutron stars (XDINSs; Turolla 2009), and Central Compact Objects (CCOs; Mereghetti 2011). The large amount of different acronyms might already show how diverse is the neutron star class, and on the other hand, how far we are from a unified scenario. These objects are amongst the most intriguing populations in modern high-energy astrophysics and in physics in general. They are precious places to test gravitational and particle physics, relativistic plasma theories, as well as strange quark states of matter and physics of atoms and molecules embedded in extremely high magnetic fields (impossible to be reproduced on Earth). Since their discovery in the late sixties, about 2000 rotational powered pulsars are known to date, thanks to numerous surveys using single dish radio antennas around the world (Parkes, Green Bank, Jodrell Bank, Arecibo), with periods ranging from about $1.5 \mathrm{~ms}$ to $12 \mathrm{~s}$ (see Figure 4, and the ATNF on-line catalog: Manchester et al. 2005), and they have magnetic fields ranging 


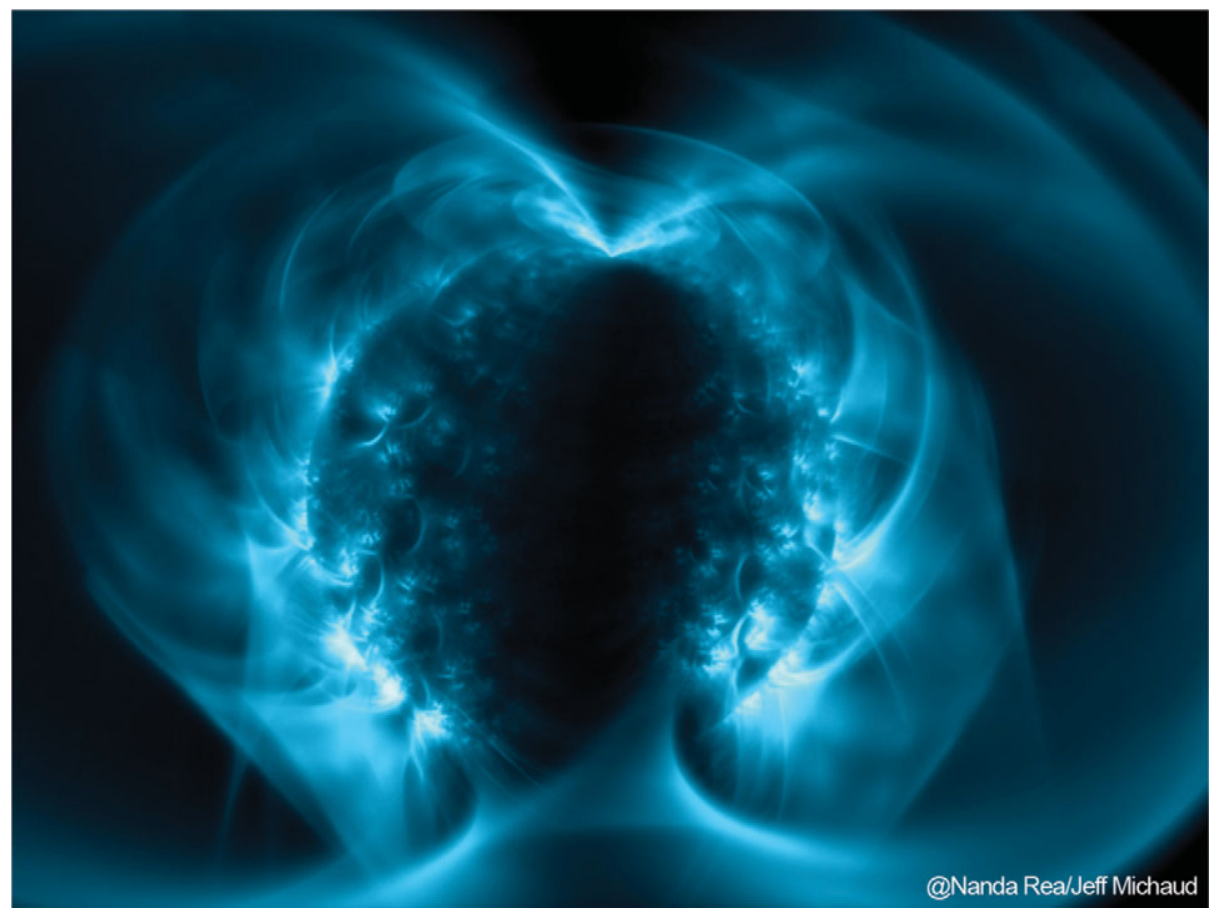

Figure 1. Artistic image of the magnetic field of a magnetar close to its surface (Rea et al. 2012; published in Science as Editors' choice).

between $\sim 10^{10}-10^{15}$ Gauss. The energy reservoir of all those pulsars is well established to be their rapid rotation, having a rotational luminosity $L_{\text {rot }} \sim 4 \pi^{2} I \dot{P} / P^{3} \sim 3.9 \times$ $10^{46} \dot{P} / P^{3} \mathrm{erg} / \mathrm{s}$. A key ingredient to activate the radio emission is the acceleration of charged particles, which are extracted from the star's surface by an electrical voltage gap. The voltage gap forms due to the presence of a dipolar magnetic field co-rotating with the pulsar, and it is believed to extend up to an altitude of $\sim 10^{4} \mathrm{~cm}$ with a potential difference $>10^{10}$ statvolts. Primary charges are accelerated by the electric field along the magnetic field lines to relativistic speeds and emit curvature radiation. Curvature photons are then converted into electron-positron pairs and this eventually leads to a pair cascade which is ultimately responsible for the coherent radio emission we observe from radio pulsars. Very energetic pulsars are also observed until the gamma-ray range, most probably in the form of synchrotron photons coming from the acceleration in the so-called 'outer-gap' of the pulsar magnetosphere (Goldreich \& Julian 1969; Ruderman $\&$ Sutherland 1975). All isolated pulsar rotational periods are increasing in time. This spin down is quantified by the braking index, $n=\Omega \ddot{\Omega} / \dot{\Omega}^{2}$ (where $\Omega=1 / P$ ). With this definition, under the assumption of pure dipole braking, we would expect all pulsars having $n=3$.

In this review we will report on the state of the art of the study of the strongest magnets in the Universe: the magnetars. However, before presenting these ultra-magnetic objects, it is instructive to indicate how the magnetic field of isolated pulsars is commonly estimated. Assuming that pulsars slow down due to magnetic dipole radiation, the surface dipolar magnetic field $\left(B_{d i p}\right)$ can be estimated from the measured pulsar spin period $\mathrm{P}$ and its first derivative $\dot{P}: B_{\text {dip }} \sim 3.2 \times 10^{19} \sqrt{P \dot{P}}$ Gauss (where $\mathrm{P}$ is in units of seconds). 


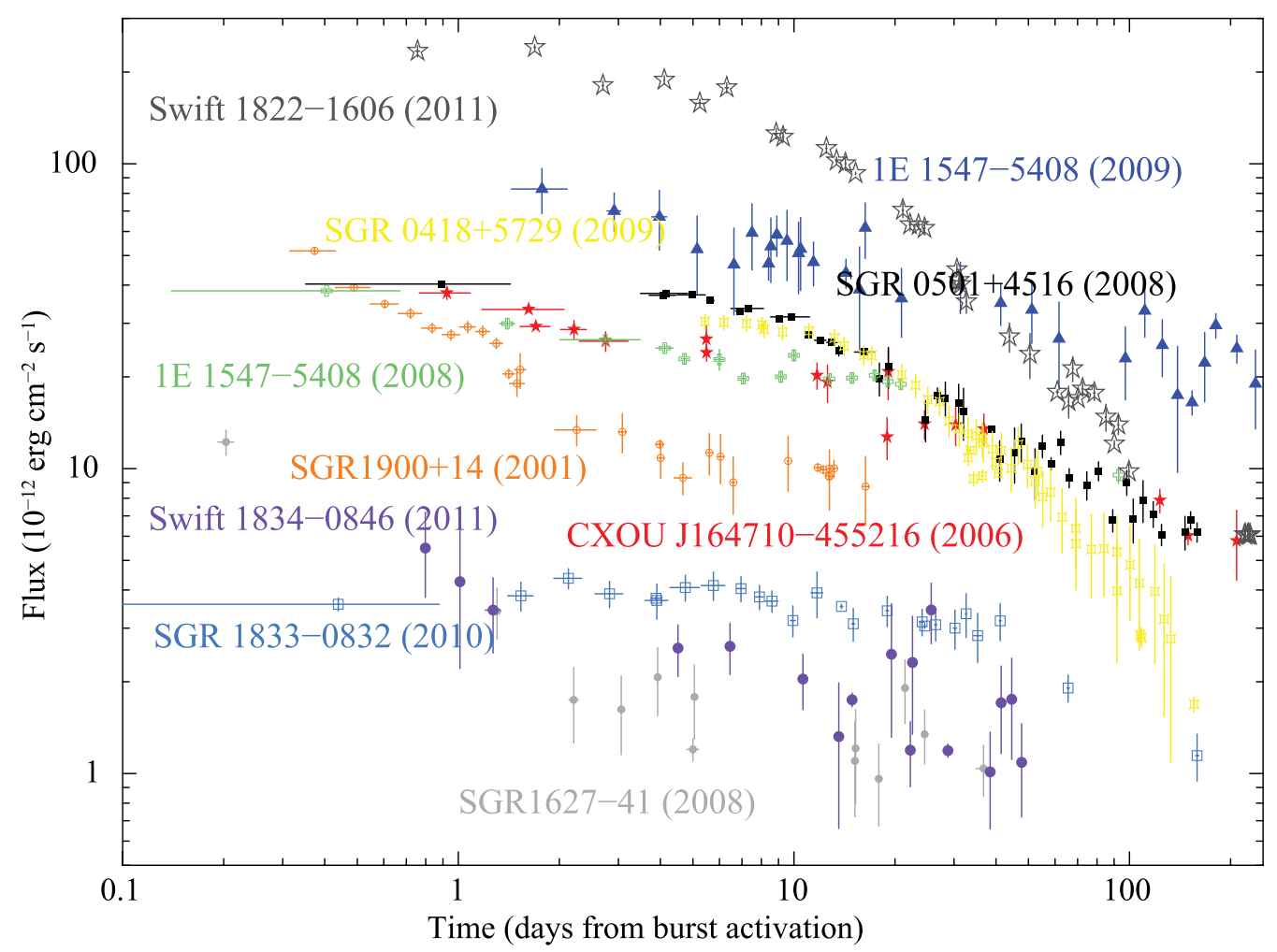

Figure 2. Flux evolution over the first $\sim 200$ days of all magnetar outbursts (only if observed with imaging instruments, and for which this period span is well monitored). Fluxes are reported in the 1-10 keV energy range, and the reported times are calculated in days from the detection of the first burst in each source. See Rea \& Esposito (2011) for the reference for each reported outburst.

The 'magnetars' (comprising AXPs and SGRs; Mereghetti 2008) are a small group of Xray pulsars (about twenty objects with spin periods between 2-12 s) the emission of which is very hardly explained by any of the scenarios for the radio pulsar or the accreting $\mathrm{X}$-ray binary populations. In fact, the very strong X-ray emission of these objects $\left(L_{x} \sim 10^{35}\right.$ $\mathrm{erg} / \mathrm{s}$ ) seemed too high and variable to be fed by the rotational energy alone (as in the radio pulsars), and no evidence for a companion star has been found so far in favor of any accretion process (as in the X-ray binary systems). Moreover, roughly assuming them being magnetic dipole radiator, their inferred magnetic fields appear to be as high as $B_{\text {dip }} \sim 10^{14}-10^{15}$ Gauss. They are then higher than the electron critical magnetic field, $B_{Q}=m_{e}^{2} c^{3} / e h \sim 4.4 \times 10^{13} \mathrm{G}$ at which an electron gyro-rotating around such magnetic field line gains a cyclotron energy equal to its rest mass. At fields higher than $\mathrm{B}_{Q}, \mathrm{QED}$ effects such as vacuum polarization or photon splitting, can take place (see Harding \& Lai 2006).

Because of these high B fields, the emission of magnetars was thought to be powered by the decay and the instability of their strong fields (Duncan \& Thompson 1992; Thompson \& Duncan 1993, 1995). This powerful X-ray output is usually well modeled by a thermal emission from the neutron star hot surface (about $3 \times 10^{6}$ Kelvin) reprocessed in a twisted magnetosphere through resonant cyclotron scattering, a process favored only under these extreme magnetic conditions (Thompson, Lyutikov \& Kulkarni 2002; Nobili, Turolla \& Zane 2008; Rea et al. 2008; see Figure 1 for an artistic representation of a magnetar). 

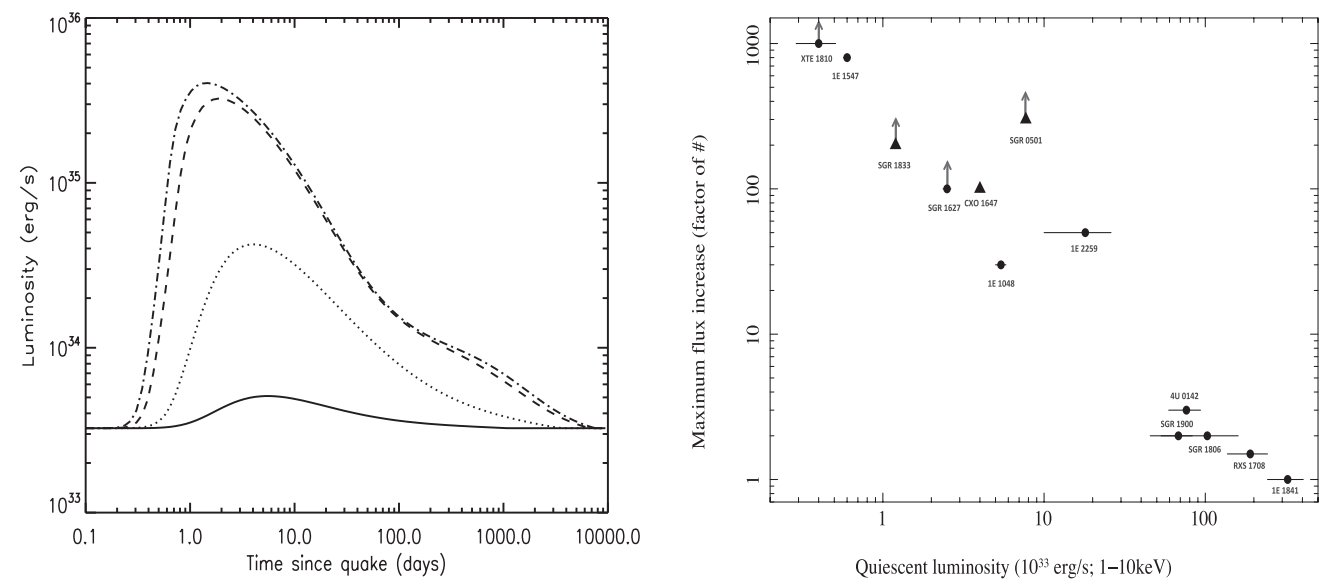

Figure 3. Left: Luminosity vs. time after energy injection. The models correspond to $E_{\text {oc }}=1.7 \times 10^{41} \mathrm{erg}$, (solid line), $1.7 \times 10^{42} \mathrm{erg}$ (dotted line), $1.7 \times 10^{43} \mathrm{erg}$ (dashed line), and $1.7 \times 10^{44} \mathrm{erg}$ (dash-dotted line). Right: quiescent luminosity vs. outburst maximum flux increase (all in the 1-10 keV band), for all magnetars showing bursts, glitches or outbursts. See Pons \& Rea 2012 for further details.

On top of their persistent X-ray emission, magnetars emit very peculiar flares on short timescales (from fraction to hundreds of seconds) emitting a large amount of energy $\left(10^{40}-10^{46} \mathrm{erg}\right.$; the most energetic Galactic events after the supernova explosions). They are probably caused by large scale rearrangements of the surface/magnetospheric field, either accompanied or triggered by fracturing of the neutron-star crust, as a sort of stellar quakes. Furthermore, magnetars also show large outbursts where their steady emission can be enhanced up to $\sim 1000$ times its quiescent level (see Figure 2, and see Rea \& Esposito 2010 for recent review on transient magnetars). From the few wellmonitored events, we are starting to understand how those outbursts are produced. They are caused by similar crustal fractures as the shorter flares, accompanied by strong surface heating, and often by the appearance of additional hot spots on the neutron-star surface. This is what may cause large spectral changes during outbursts, pulse profile variability, and different cooling patterns depending on the outburst. We have recently started to model that outburst decay, and much important physical information is slowly emerging, i.e. that all outbursts saturate at $\sim 10^{36} \mathrm{erg} / \mathrm{s}$, due to neutrino cooling processes, and regardless the source quiescent level. This discovery makes magnetar outbursts potential standard candles (Pons \& Rea 2012; see Figure 3).

\section{Hints for the connection between magnetars and radio pulsars}

In the past few years, new discoveries started to shed light on a possible connection between magnetars and the typical radio pulsar population, weakening the strong distinction between these two classes, while pointing to a continuum of magnetar-like emission in the neutron star population. Below we list a few of those key discoveries.

- Magnetars were believed to be radio-quiet sources for a few decades. This was interpreted as the result of a photon splitting process that is very efficient in magnetic fields stronger than the critical electron field $\left(B_{Q}\right.$; Baring 1998). The discovery in 2004 of transient magnetars coincided also with the discovery of radio pulsed emission from such sources (Camilo et al.2006; Levin et al. 2010). Magnetar pulsed radio emission, however, appeared to have different properties with respect to normal radio pulsars (flat radio 


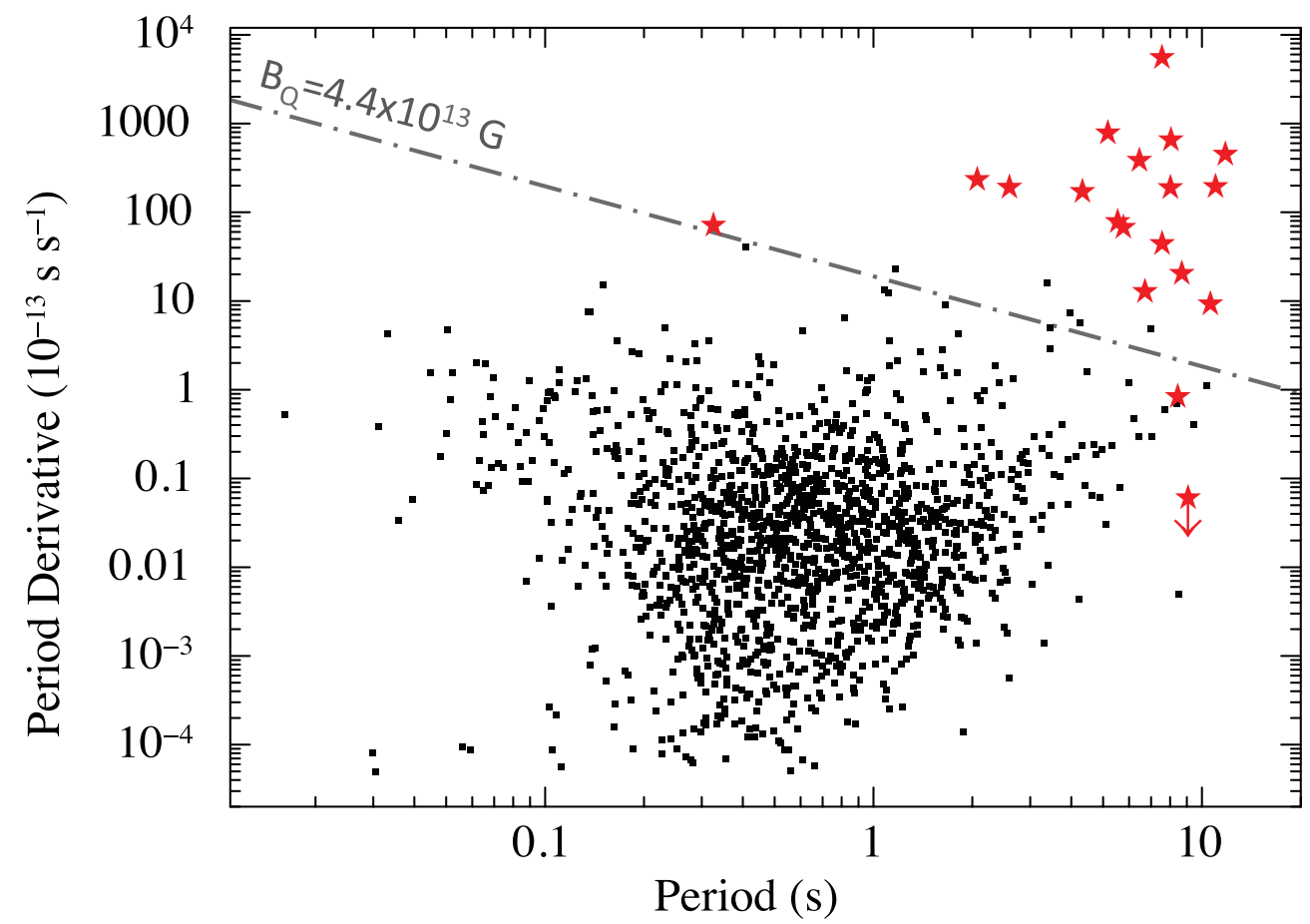

Figure 4. $P-\dot{P}$ diagram for all known isolated pulsars. Black squares represent normal radio pulsars, and red stars are all pulsars showing magnetar-like emission. The two newly discovered low-B magnetars: Swift J1822.3-1606 (Rea et al. 2012), and SGR 0418+5729 (Rea et al. 2010) are also reported, as well as the electron quantum magnetic field (dash-dotted grey line).

spectra, large variability, connection with X-ray outbursts). This came as a big surprise, and started the idea of a possible connection between magnetars and the typical radio pulsars. Furthermore, recently a study of radio magnetars showed that despite the different characteristics, the radio emission can have the same physical mechanisms as for rotation-powered pulsars (Rea et al. 2012): powered by rotational energy (see also Figure 5 ), but with different observational properties possibly caused by a different path that a pair cascade might undertake when embedded in a mostly toroidal magnetic field.

- Deep radio surveys discovered a few radio pulsars having dipolar fields larger than the $B_{Q}$ (see Figure 4). Although having magnetic fields in the magnetar range those objects were behaving as normal radio pulsars, and this was interpreted by a different magnetic geometry between the two classes. In 2008, bursting activity and an X-ray outburst were detected from a high-B pulsar, showing the presence of magnetar-like activity (Gavriil et al. 2008; Kumar \& Safi-Harb 2008).

- The extensive follow-up of transient magnetars undergoing an outburst had allowed the most unexpected discoveries. In particular, prompted by detection of typical magnetar-like bursts and a powerful outburst of the persistent emission, a new transient magnetar was discovered in 2009, namely SGR 0418+5729. However, with great surprise after more than a year of extensive monitoring, no period derivative was detected, which led to an upper limit on the source surface dipolar field of $B_{d i p}<7.5 \times 10^{12}$ Gauss (Rea et al. 2010). For the first time we witnessed a magnetar with a low dipolar magnetic field. This discovery demonstrated that not only a critical magnetic field $\left(>B_{Q}\right)$ was not necessary to have magnetar-like activity, but many 'apparently' normal pulsars can 


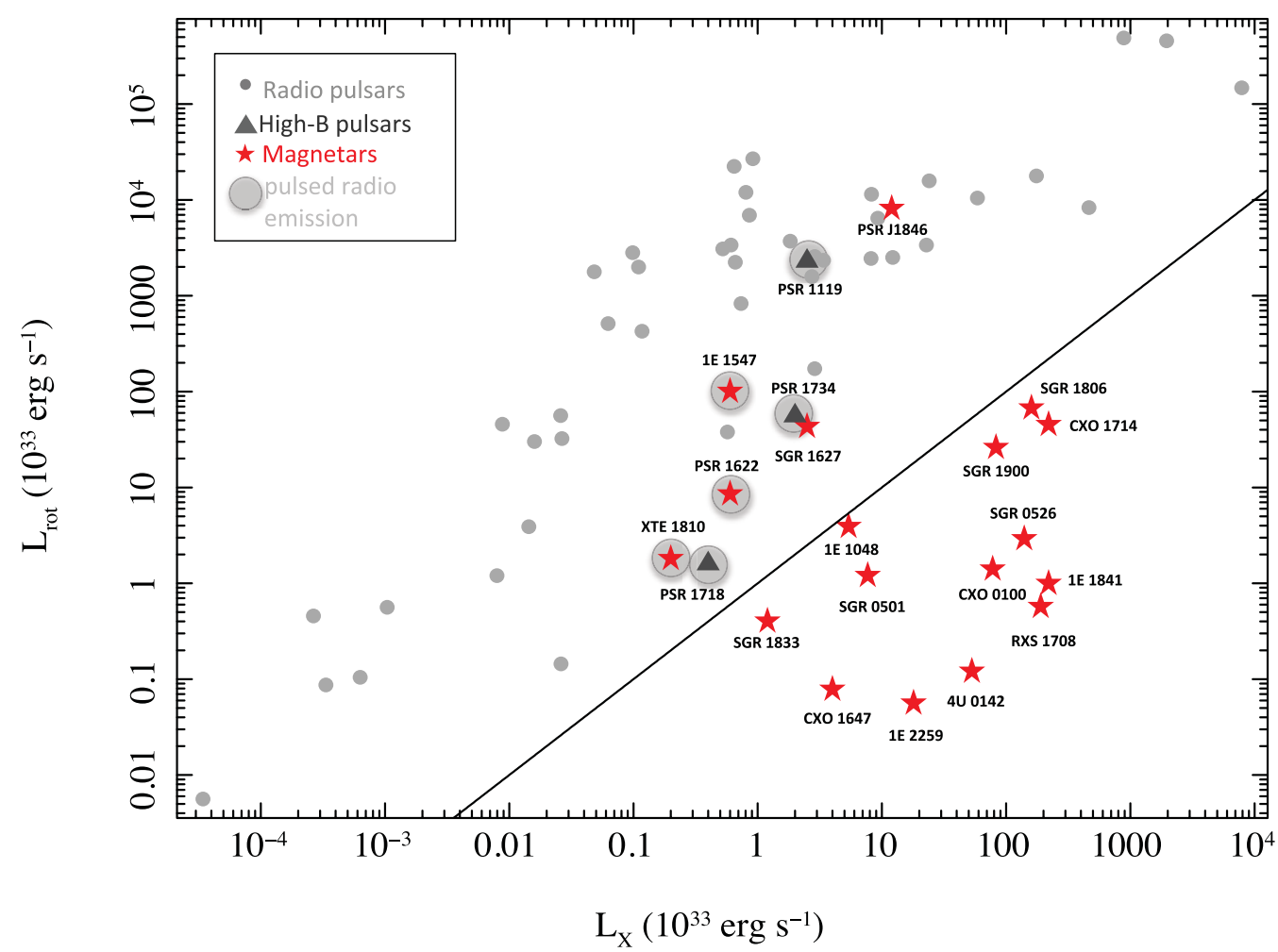

Figure 5. X-ray luminosity versus the spin-down luminosity for all pulsars having a detected X-ray emission (grey filled circles), high-B pulsars (filled triangle), and the magnetars (red stars). Grey shaded circles mark the magnetars and high-B pulsars with detected pulsed radio emission, and the solid line shows $L_{x}=L_{\text {rot }}$. X-ray luminosities are calculated in the $0.5-10 \mathrm{keV}$ energy range, and for variable sources refer to the quiescent emission state.

turn out as magnetars at anytime (in fact the discovery of a second low-B magnetar soon followed; Rea et al. 2012; Scholz et al. 2012; see also Figure 4).

- Advances in the measurement of pulsar breaking indexes showed the existence of objects with indexes $n$ smaller than 3 , which would imply an increasing magnetic field with age under the common magnetic breaking picture. In particular this is the case of the high-B pulsar PSR 1734-33 (Espinoza et al. 2011), discovered to have $n=0.9$. This discovery favors models for which the magnetic field is buried into the crust by accretion in the first supernova phases, and start re-emerging during the pulsar life-time (Viganó \& Pons 2012). A similar conclusion has been reached with the discovery of low-B fields in CCOs, whose young age and hot surface temperature are instead pointing to a strong buried magnetic field, despite what measured by their period and period derivatives (Halpern et al. 2007).

\section{Conclusions}

The above discoveries, among others, re-focus the attention on a few important ingredients of neutron star physics: i) the surface dipolar magnetic field strength cannot be the only parameter driving their magnetar or radio pulsar nature, ii) magnetars can behave as radio pulsars and vice-versa, possibly powered by a similar mechanism sustained by 
rotational energy, and iii) an internal strong magnetic field is required to explain the low braking indexes of a few radio pulsars, as well as the emission of the compact central objects, despite the rather low dipolar magnetic field component.

These discoveries show that extremely strong magnetic fields may be very common among the pulsar population, rather than an exception. This might imply that supernova explosions should be generally able to produce such strong magnetic fields, hence that most massive stars are either producing fast rotating cores during the explosion to activate the dynamo, or are strongly magnetized themselves (i.e. 1 kGauss at least). Furthermore, in this scenario several gamma-ray bursts (not only an irrelevant fraction), might indeed be due to the formation of magnetars, and the gravitational wave background produced by magnetar formation should then be larger than predicted so far (important for future instruments as Advanced-LIGO).

\section{Acknowledgements}

NR is supported by a Ramón y Cajal research position to CSIC, as well as by grants AYA2009-07391, SGR2009-811, TW2010005 and iLINK 2011-0303. She is indebted to José Pons, Gian Luca Israel, Paolo Esposito, Roberto Turolla, Silvia Zane, Daniele Viganó, Rosalba Perna, Diego Torres, and to many others, for the always exciting and pleasant time spent together to understand the physics behind the bewildering magnetar emission. NR wishes also to thank Dick Manchester, all the speakers, and the IAU organizers for the excellent meeting.

\section{References}

Baade, W. \& Zwicky, F. 1934, Physical Review 46, 76-77

Baring, M. G. \& Harding, A. K., 1998, ApJ, 507, L55

Camilo, F., Ransom, S. M., Halpern, J. P., Reynolds, J., Helfand, D. J., Zimmerman, N., \& Sarkissian, J. 2006, Nature 442, 892

Chandrasekhar, S. 1931, ApJ 74, 81

Duncan, R. C. \& Thompson, C 1992, ApJ 392, L9-L13

Espinoza, C. M., Lyne, A. G., Kramer, M., Manchester, R. N., \& Kaspi, V. M., 2011, ApJ 741, L13

Gavriil, F. P., Gonzalez, M. E., Gotthelf, E. V., Kaspi, V. M., Livingstone, M. A., \& Woods, P. M. 2008, Science 319, 1802-1805

Goldreich, P. \& Julian, W. H., 1969, ApJ,157, 869

Harding, A. K. \& Lai, D. 2006, RPPh, 69, 2631

Hewish, A., Bell, S. J., Pilkington, J. D. H., Scott, P. F., \& Collins, R. A. 1968, Nature 217, 709 Halpern, J. P., Gotthelf, E. V., Camilo, F., \& Seward, F. D. 2007, ApJ 6651304

Keane, E. F. \& McLaughlin, M. A. 2011, BASI, 39, 333

Kumar, H. S. \& Safi-Harb, S. 2008, ApJ 678, L43-L46

Levin, L., Bailes, M., Bates, S., Bhat, N. D. R., Burgay, M., Burke-Spolaor, S., D'Amico, N., Johnston, S., Keith, M., Kramer, M., Milia, S., Possenti, A., Rea, N., Stappers \& B., van Straten, W. 2010, ApJ 721, L33-L37

Manchester, R. N., Hobbs, G. B., Teoh, A., \& Hobbs, M. 2005, AJ 129, 1993

Mereghetti, S. 2008, A\&AR, 15225

Mereghetti, S. 2011, APSS, "High-Energy Emission from Pulsars and their Systems", Eds. N. Rea \& D. F. Torres, Springer-Verlag Berlin

Nobili, L., Turolla, R., \& Zane, S. 2008, MNRAS, 386, 1527

Pons, J. A. \& Rea, N. 2011, ApJ 750, L6

Rea, N., Zane, S., Lyutikov, M., \& Turolla, R. 2007, ApESSS 308, 61 
Rea, N., Zane, S., Turolla, R., Lyutikov, M., \& Götz, D. 2008, ApJ 686, 1245

Rea, N., Esposito, P., Turolla, R., Israel, G. L., Zane, S., Stella, L., Mereghetti, S., Tiengo, A., Gotz, D., Gogus, E., \& Kouveliotou, C. 2010, Science 330, 944

Rea, N. \& Esposito, P. 2011, APSS, "High-Energy Emission from Pulsars and their Systems", Eds. N. Rea \& D. F. Torres, Springer-Verlag Berlin

Rea, N., Pons, J. A., Torres, D. F., \& Turolla, R., 2012, ApJ, 748, L12

Rea, N., Israel, G. L., Esposito, P., Pons, J. A., Camero-Arranz, A., Mignani, R. P., Turolla, R., Zane, S., Burgay, M., Possenti, A, et al. 2012, ApJ 754, 27

Ruderman, M. A. \& Sutherland, P. G. 1975, ApJ, 196, 51

Scholz, P., Ng, C.-Y., Livingstone, M. A. et al. 2012, arXiv:1204.1034

Thompson, C., Lyutikov M. \& Kulkarni S. R. 2002, ApJ 574, 332

Thompson, C. \& Duncan, R. C. 1993, ApJ 408, 194

Turolla, R. 2009, ASSL, 357, 141

Vigano, D. \& Pons, J. A. 2012, MNRAS, 425, 2487 